\title{
Incontinencia y trastornos miccionales: ¿qué podemos hacer?
}

\author{
MÁ. San José González $z^{\mathrm{a}}$ P. Méndez Fernández \\ aPediatra. Servicio de Atención Primaria. Sarria, Lugo. España. \\ ${ }^{\circ}$ Pediatra. Servicio de Atención Primaria Illas Canarias. Servizo Galego de Saúde. \\ Gerencia de Atención Primaria. Lugo. España.
}

Rev Pediatr Aten Primaria. 2009; I :el-e29

Miguel Ángel San José González, miguel.san.jose.gonzalez@sergas.es

Resumen

Las alteraciones relativas a la micción son un motivo de consulta cada vez más frecuente en pediatría. Pueden referirse tanto a problemas del patrón miccional diurno como a incontinencia. Las infecciones urinarias de repetición también pueden ser una expresión de estos trastornos. Pueden deberse a la presencia de anomalías orgánicas, como la obstrucción anatómica del tracto urinario inferior o la vejiga neurógena. Pero son más frecuentes las causas funcionales, que clásicamente se han agrupado bajo el término micción disfuncional y que en la actualidad se denominan trastornos de la función del tracto urinario inferior. Su repercusión clínica varía desde los cambios en el patrón miccional o la incontinencia leve hasta trastornos más graves que llevan a la alteración irreversible de la función vesical. El diagnóstico descansa fundamentalmente en una buena historia clínica y en la exploración física junto con el llamado diario miccional. Las exploraciones complementarias (incluido el examen urodinámico) han de valorarse individualmente.

Una comprensión adecuada del funcionamiento normal y anormal de la vejiga permite un abordaje satisfactorio del problema desde la Pediatría de Atención Primaria. Hay que dar la mayor importancia al primer nivel de tratamiento: hábitos dietéticos sanos, tratamiento del estreñimiento, higiene correcta y patrón miccional regular. Cuando la evaluación inicial identifica un problema orgánico, o cuando el tratamiento inicial fracasa, es necesario derivar al nivel hospitalario.

Palabras clave: Trastorno miccional, Incontinencia, Micción disfuncional.

Abstract

Alterations on urination are a common reason for more and more frequent consultation in Pediatrics. They can refer so much to problems of daytime voiding pattern as to incontinence. Recurrent urinary tract infections can also be an expression of these disorders. They can be due to the presence of organic anomalies such as the anatomical obstruction of the lower urinary tract or the neurogenic bladder. But there are more common the functional causes, which tradi-

Los autores declaran no presentar posibles conflictos de intereses en relación con la preparación y publicación de este artículo. 
tionally have been grouped under the term dysfunctional voiding and at present they are named disorders of the lower urinary tract function. Its clinical impact varies from changes in the pattern of voiding or slight incontinence, to more severe disorders that lead to irreversible alteration of bladder function. The diagnosis rests fundamentally on a good clinical history and physical examination along with the so-called voiding diary. The complementary explorations (including urodynamic evaluation) should be assessed individually.

A suitable understanding of normal and abnormal functioning of the bladder allows a satisfactory approach to the problem in the paediatric primary care. We must give great importance to the first level of treatment: healthy diet, treatment of constipation, proper hygiene and regular voiding pattern. When the initial evaluation identifies an organic problem, or when initial treatment fails, we must refer the hospital level.

Key words: Voiding disorders, Incontinence, Voiding dysfunction.

\section{Introducción}

En los últimos años, las alteraciones relativas a la micción han ganado progresivamente una mayor importancia, no sólo por su incidencia, sino también por sus posibles repercusiones patológicas y/o psicológicas (personales y familiares). Pueden referirse tanto a problemas del hábito miccional diurno como a incontinencia. En su etiología pueden participar anomalías orgánicas, como la obstrucción anatómica del tracto urinario inferior o la vejiga neurógena, pero son más frecuentes las causas funcionales, que clásicamente se han agrupado bajo términos como micción disfuncional o trastornos miccionales no neurógenos, y que en la actualidad se denominan trastornos de la función del tracto urinario inferior.

Aunque no existen datos epidemiológicos que nos informen adecuadamente sobre la prevalencia, algunos estudios lle- vados a cabo mediante cuestionarios, en escolares sanos de 4 o más años de edad, muestran incontinencia diurna en el 3$6 \%$ de los niños y síntomas miccionales en el $20-26 \%{ }^{1-3}$. La frecuencia disminuye con la edad y aunque pueden presentarse en ambos sexos, se considera que son más importantes en niñas preescolares y escolares ${ }^{2,4}$. Algunas publicaciones citan los trastornos miccionales como un motivo frecuente de consulta en pediatría pero, en general, se considera que son un problema infradiagnosticado. La edad, el contexto y la historia clínica pueden modificar la impresión de patología o normalidad de muchos de los síntomas propios de los trastornos miccionales (por ejemplo, la incontinencia diurna puede considerarse normal en niños con edades inferiores a los límites habituales para el entrenamiento de esfínteres, o en situaciones de excesiva ingesta líquida o de estrés emocional). 
En los niños que controlan sus esfínteres, hay una relación estrecha entre infecciones del tracto urinario (ITU), reflujo vesicoureteral (RVU), estreñimiento y trastornos miccionales. Muchos autores utilizan la denominación de síndrome de eliminación disfuncional para resaltar la frecuente asociación entre el estreñimiento y los trastornos miccionales 5 . La retención de heces en el recto repercute negativamente sobre la función vesical y sobre la dinámica del suelo pélvico durante la micción ${ }^{6}$. Un estudio retrospectivo basado en controles de salud de niños de 4-17 años (a los que se preguntaba por la presencia o no de problemas en sus hábitos de eliminación), demostraba cómo la prevalencia de incontinencia fecal y urinaria era mayor en los niños con estreñimiento al compararlos con los que no padecían este problema $^{7}$. En niños controlados por infecciones de orina recurrentes o por incontinencia urinaria, el tratamiento del estreñimiento (que muchas veces no reconocen inicialmente como molestia los propios padres o los niños) mejora o elimina el problema por el que siguen consulta ${ }^{6}$.

También se ha demostrado la asociación de las infecciones de orina recurrentes y los trastornos miccionales, sobre todo en niñas y cuando el primer episodio de ITU se diagnostica a partir de los tres años de edad ${ }^{8}$. Por un lado, es más probable encontrar anomalías miccionales cuando se estudia a los niños con antecedente de ITU recurrentes (hasta el $80 \%$ de los niños con ITU recurrentes tienen síntomas miccionales); $y$, por otro, un $70-80 \%$ de los niños controlados por algún problema miccional tiene antecedente de ITU ${ }^{10,11}$.

El estreñimiento y los trastornos miccionales no sólo son un factor de riesgo para desarrollar ITU (sobre todo recurrentes), sino que también están relacionados con el RVU. Hasta un $46 \%$ de los niños estudiados en el nivel hospitalario por una alteración miccional (vejiga hiperactiva o micción disfuncional) pueden tener también RVU y ésta a menudo suele ser de grado III o mayor (sobre todo en los de menor edad) ${ }^{12}$. En este estudio de Z. Ural et al. también encontraron, en el grupo de niños con antecedente de ITU, asociación entre el trastorno miccional con RVU y la probabilidad de hallar anomalías corticales renales (45\% frente a $17 \%$ en el grupo con ITU pero $\sin \mathrm{RVU}, \mathrm{p}<0,001)$.

Además, los trastornos de la función del tracto urinario inferior pueden contribuir a enlentecer la resolución del $\mathrm{RVU}^{6}$. Diversos trabajos muestran un menor porcentaje de resolución de los 
reflujos de grado mayor de III en el grupo con alteración de la función vesical, así como la alta incidencia de inestabilidad vesical en los casos de RVU bilateral, o el fracaso de la cirugía antirreflujo en este tipo de pacientes y la resolución significativa del RVU cuando se pauta un tratamiento con anticolinérgicos ${ }^{13-15}$.

\section{Micción normal}

Para entender y enfocar adecuadamente el problema de la incontinencia y los trastornos miccionales es útil una breve descripción de la secuencia normal de la micción y del proceso madurativo que lleva a la continencia.

\section{Micción normal}

La secuencia normal de una micción comprende las fases de llenado y vaciamiento (tabla I). Durante la fase de llenado, la vejiga se acomoda a los volúmenes crecientes de orina con una presión intravesical baja, y las vías inhibitorias del sistema nervioso central (SNC) impiden el vaciado de la vejiga hasta que llegue un momento apropiado: inhiben las contracciones vesicales involuntarias (músculo detrusor) y, además, mantienen cerrado el tracto de salida (cuello vesical y esfínter urinario). Durante la fase de vaciado, de un modo coordinado se contrae el músculo detrusor y se relajan tanto la musculatura pélvica (el cuello vesical desciende) como el esfínter uretral.

¿Cómo es el control diurno maduro? A partir de cierto volumen de llenado aparece la sensación miccional. En ese momento, el niño puede decidir entre retener u orinar. Si decide retener, la vejiga se acomoda a su contenido (se inhibe la actividad del detrusor) y la sensación de micción puede desaparecer.

Tabla I. Fases de una micción normal

\begin{tabular}{|c|c|c|}
\hline & Fase de llenado & Fase de vaciamiento \\
\hline Predominio nervioso & Simpático & Parasimpático \\
\hline Actividad del esfínter externo & $\uparrow \uparrow \uparrow$ & Inhibida \\
\hline Volumen vesical & Aumento progresivo & Descenso hasta 0 \\
\hline Actividad del músculo detrusor & Inhibida por el SNC & $\uparrow \uparrow$ \\
\hline Presión vesical & Baja a pesar del llenado & Aumento moderado \\
\hline Presión del cuello vesical & $\uparrow \uparrow \uparrow$ & $\downarrow \downarrow$ \\
\hline \multicolumn{3}{|c|}{$\begin{array}{l}\text { SNC: sistema nervioso central. } \\
\text { Cada una de estas dos fases se caracteriza por la adaptación del tracto urinario inferior a dos funciones opuestas } \\
\text { llenado de un volumen adecuado de orina con baja presión, sin fugas, y vaciamiento (micción) eficiente, volun } \\
\text { tario, completo y con baja presión. La capacidad vesical normal puede estimarse con la fórmula (en mililitros) } \\
\text { volumen }=30 \text { x [edad (en años) }+2 \text { ]. }\end{array}$} \\
\hline
\end{tabular}


Posteriormente, con un mayor volumen de orina, aparece una sensación más apremiante, que ya no se puede inhibir completamente, pero se puede retener la orina (aunque persista el deseo miccional) hasta que socialmente se encuentran el lugar y el momento apropiados para orinar. Durante la micción consciente, el esfínter externo se relaja completamente antes de contraerse la vejiga. La relajación del esfínter y la contracción vesical han de ser coordinadas para un vaciado adecuado ${ }^{16}$.

\section{Maduración de la micción}

En el período neonatal, la micción es refleja y está controlada por los centros espinales. El neonato orina unas 20 veces al día, frecuencia que presenta una ligera disminución a lo largo del primer año de vida. Con el crecimiento, la capacidad vesical aumenta y el niño orina menos a menudo. En el segundo año de vida suele iniciarse el proceso que llevará al control. Esta etapa durante la cual el niño madura hasta adquirir el control vesical maduro, pasando desde la función infantil del tracto urinario inferior (involuntaria-refleja) a la función adulta (consciente-voluntaria), es denominada fase de transición. En primer lugar, se adquiere la conciencia de vejiga llena $y$, a continuación, entre los dos y los tres años de edad, se desarrolla la capacidad para iniciar o inhibir la micción de modo voluntario. Pero el verdadero control voluntario (patrón adulto o maduro de micción) no se alcanza, en general, hasta los cuatro años. Inicialmente el niño tiene un mejor control sobre el esfínter que sobre la vejiga, por ello le resulta más fácil detener la micción que iniciarla. Pudiera ocurrir que para detener una micción se realizara la contracción del esfínter en lugar de inhibir la contracción vesical. La persistencia de este patrón puede originar disinergia esfínter-vejiga ${ }^{17}$.

\section{Entrenamiento de esfínteres}

La mayoría de los niños desarrolla una funcionalidad y una continencia urinarias normales independientemente del momento y el tipo de entrenamiento miccional que se utilice. Sin embargo, en algunos casos, el retraso o un inadecuado entrenamiento de esfínteres pueden favorecer el desarrollo de un trastorno de la función del tracto urinario inferior. Aunque no hay evidencias concluyentes, se admite que los siguientes consejos para la educación de esfínteres pueden ser beneficiosos para alcanzar la continencia urinaria diurna más temprana y evitar los trastornos miccionales ${ }^{18}$.

- Iniciar la enseñanza de la continencia urinaria alrededor de los 18 me- 
ses de edad, cuando se espacia el período de pañal seco (por ejemplo, cuando el niño consigue con frecuencia no orinarse durante la siesta) y se ha desarrollado la conciencia de vejiga llena.

- Facilitar el trabajo de la musculatura pélvica durante la micción usando un orinal o una taza de váter adaptada en la que el niño apoye bien tanto las nalgas como los pies.

- Favorecer el vaciamiento vesical completo y la coordinación esfínter-vejiga animando al niño a que orine cuando se le vea o suponga con ganas (para que lo logre al primer intento) y con un intervalo adecuado con respecto a la micción previa (de dos a cuatro horas según la edad y la ingesta de líquidos). No mantener al niño sentado en el orinal hasta que logre la micción y no insistir en que haga esfuerzo si fracasa el primer intento.

- No dispersar los esfuerzos cambiando de técnica continuamente y ser persistentes en esta educación, ya que se logra el objetivo en menos de tres meses.

\section{Incontinencia y trastornos funcionales}

El campo de la urología es un terreno de frecuente anarquía semántica. Dife- rentes grupos y autores utilizan desiguales definiciones para los mismos términos (como enuresis, incontinencia, vejiga hiperactiva o micción disfuncional), creando confusión. En julio de 2006, la International Children's Continence Society (ICCS) publicó un informe para estandarizar la terminología ${ }^{19}$. Las definiciones y términos que mencionemos a lo largo de esta revisión se ajustarán a este informe.

Las alteraciones funcionales del tracto urinario inferior pueden manifestarse por cambios en el patrón miccional (por ejemplo, urgencia, esfuerzo o cambios en la frecuencia), por pérdidas de orina o por infecciones urinarias recurrentes. La incontinencia diurna a menudo está entrelazada con los síntomas miccionales, pero la incontinencia no es obligada, es decir, no todos los que tienen alteraciones funcionales son incontinentes. $\mathrm{Y}$ a la inversa, no toda pérdida de orina intermitente diurna se debe a un trastorno funcional. Aclaremos conceptos:

\section{Incontinencia}

La incontinencia urinaria es la pérdida incontrolable de orina. Puede ser continua 0 intermitente. La incontinencia continua supone la pérdida constante de orina, hecho que es 
siempre patológico (independientemente de la edad) y que se debe a malformaciones congénitas (uréter ectópico, por ejemplo) o a daño iatrogénico sobre el esfínter uretral externo. La incontinencia intermitente es la pérdida involuntaria de orina, de modo discontinuo (con períodos secos) y después de la edad a la que se suele conseguir controlar la orina. Puede ser diurna o nocturna. Se reserva el término enuresis para la incontinencia intermitente que aparece durante el sueño por la noche (enuresis nocturna). El término enuresis diurna ha quedado obsoleto y debe evitarse; es preferible hablar de incontinencia diurna (intermitente). Si el niño moja tanto de día como de noche, han de nombrarse tanto la incontinencia diurna como la nocturna o enuresis. Enuresis es sinónimo de incontinencia intermitente nocturna.

\section{Incontinencia diurna}

Hay una etapa de la vida en la que las pérdidas intermitentes e involuntarias de orina son normales (primeros años y fase de transición). La edad de cinco años marca el límite de esta fase, es decir, toda pérdida diurna a partir de dicha edad (a veces antes, según las circunstancias) ha de ser tenida en cuenta desde un punto de vista clínico. Es un problema habitual que afecta por igual a los dos sexos y cuya frecuencia disminuye con la edad: un $8-10 \%$ de los niños de más de cinco años tiene pérdidas de orina durante el día; a los siete años un 2-3\% de los niños (en esta etapa hay una pequeña diferencia por sexo: tiene escapes un 3-4\% de las niñas) y menos de un $1 \%$ a partir de los doce años ${ }^{16}$.

La incontinencia intermitente diurna a menudo se considera una molestia inevitable y se tolera hasta que, con el inicio de la escolarización, el niño se retrasa con respecto a sus pares. En ese momento, la preocupación de los padres es habitual; pero no menos frecuente, e incluso más importante, es la repercusión en el niño (psicológica y social). Mojar la ropa es la tercera causa de estrés, según citan los propios niños al preguntarles por veinte problemas que les pueden preocupar en su etapa escolar ${ }^{20}$.

Las causas de dicha incontinencia son variadas y pueden clasificarse como neurógenas o no neurógenas (tabla II). Las más frecuentes son las no neurógenas $y$, dentro de este grupo, las funcionales (vejiga hiperactiva, por ejemplo), seguidas por las incontinencias clasificadas como irritativo-infecciosas. 
Tabla II. Causas de la incontinencia urinaria diurna y de los trastornos miccionales en la infancia

Neurógenas: anomalías de la médula espinal

- Defectos del tubo neural (disrrafias)

- Otras: traumatismos, mielitis transversa, tumor espinal

\section{No neurógenas}

- Anatómicas

- Por obstrucción de la salida vesical: válvulas de uretra posterior

- Uréter ectópico y fístula

- Anomalías del esfínter y cuello vesical: epispadias, extrofia vesical, anomalías del seno urogenital (uretra corta)

- Funcionales: trastornos de la función del tracto urinario inferior

- Vejiga hiperactiva

- Micción demorada o incontinencia por aplazamiento de la micción

- Micción disfuncional

- Vejiga hipoactiva

- Otros: incontinencia de esfuerzo, reflujo vaginal, incontinencia con la risa y frecuencia urinaria diurna excesiva

- Infección, irritación

- Infección urinaria

- Parasitaciones (oxiuros)

- Vulvovaginitis, uretritis irritativas: cuerpo extraño, trauma, abuso sexual, químicas

- Otras: hipercalciuria

Podemos establecer dos grandes grupos: neurógenas y no neurógenas. Dentro de las primeras no consideramos las anomalías del SNC que impiden o dificultan el control voluntario de esfínteres (la incontinencia es una consecuencia más de la enfermedad). Dentro de las no neurógenas tampoco mencionamos aquellas enfermedades que cursan con poliuria (insuficiencia renal crónica, diabetes mellitus, diabetes insípida), ya que no tiene por qué haber incontinencia una vez alcanzada la fase de control de esfínteres.

\section{Trastornos funcionales del tracto urinario inferior}

Son el conjunto de trastornos que tienen su origen en una disfunción vesicouretral de la fase de llenado y/o vaciado, sin una causa médica o anatómica detectable y que se presentan una vez pasada la edad de entrenamiento de esfínteres. Con frecuencia se ha empleado el término micción disfuncional (del inglés dysfunctional voiding) para referirse a este grupo de alteraciones. Sin em- bargo, el informe de la ICCS aconseja reservar dicha expresión únicamente para referirse a un tipo de patología (aquella en la que los síntomas se deben a la alteración durante la fase de vaciado vesical por la contracción inadecuada del esfínter uretral). Cuando hablamos de los trastornos en general, es preferible usar la expresión trastornos de la función del tracto urinario inferior. Como ya se ha citado, son trastornos frecuentes tanto de modo aislado, como en el con- 
texto de ITU recurrente. No obstante, a menudo pasan desapercibidos si no se pregunta específicamente por ellos.

Los trastornos miccionales funcionales son más frecuentes en niñas (relación 9/1 con respecto a los niños) y en la etapa preescolar y escolar (4-7 años de edad) ${ }^{11}$. Cuando originan molestias (por ejemplo, pérdidas de orina o cambios en el patrón miccional, como urgencia o aumento de la frecuencia urinaria), son un motivo importante de preocupación y de estrés para la vida social y familiar del paciente. Se distinguen diferentes entidades dentro de este grupo de trastornos (tabla II). La vejiga hiperactiva (a veces también llamada vejiga no inhibida o inestable) es el cuadro más frecuente, tanto clínicamente como en las series de estudios urodinámicos ${ }^{21}$. Le siguen en frecuencia la micción disfuncional y la vejiga hipoactiva o perezosa.

\section{Etiología}

No hay una causa única y definida. Influyen diferentes circunstancias, de modo que han de ser contemplados como un problema multifactorial en el que están involucrados no sólo múltiples sistemas (el sistema genitourinario, el gastrointestinal inferior y el sistema nervioso, tanto periférico, como central), sino también diferentes factores ${ }^{22}$ :
- Hereditarios: hay casos de familias con predisposición hereditaria para la enuresis nocturna e incontinencia diurna asociadas ${ }^{21}$. También se han recogido casos graves de trastornos miccionales, como el síndrome de Ochoa (vejiga neurógena no neurógena con inversión de la expresión facial) con herencia autosómica recesiva ${ }^{22}$.

- Madurativos: se refieren al retraso en la adquisición del control del SNC para regular la micción con un patrón adulto, bien por factores individuales o por factores sociales que impidan o dificulten un adecuado aprendizaje del control de esfínteres. Puede influir el retraso en el entrenamiento de esfínteres ${ }^{23}$ 0 , al contrario, un control excesivamente temprano e incompleto ${ }^{17}$.

- Conductuales: diversos autores relacionan los problemas de conducta y comportamiento con la presencia de alteraciones miccionales, aunque sin poder establecer si son causa o consecuencia ${ }^{22}$. Los niños con síntomas miccionales (comparados con los que no presentan ninguna alteración miccional) tienen con más frecuencia trastorno por déficit de atención e hiperactividad (TDAH) y otros problemas de conducta $y$ 
comportamiento (por ejemplo, trastorno de oposición desafiante) $)^{20,24}$. También se ha relacionado la obesidad con una mayor prevalencia de eliminación disfuncional (refiriéndose a la mayor frecuencia de estreñimiento y de alteraciones miccionales en niños obesos) y con una menor eficacia del tratamiento de estos problemas al tener en cuenta el índice de masa corporal de los niños que los padecen ${ }^{25,26}$.

- Irritativos: en este grupo se incluyen aquellos agentes que pueden motivar dolor o irritación de la mucosa intestinal o genitourinaria. Son situaciones que originan dolor y aparecen de modo episódico durante el período de entrenamiento de esfínteres, dificultando que el proceso normal se complete y dando lugar al llamado ciclo de la eliminación disfuncional ${ }^{18}$. Por ejemplo, una infección de orina, una uretritis (balanitis, vulvovaginitis) o un estreñimiento agudo producen molestias en la zona perineal, ocasionando una peor relajación del esfínter uretral durante la micción. Esto aumenta el riesgo de residuo miccional o disminuye la frecuencia de micciones, lo que, a su vez, aumenta el riesgo de nuevas infecciones o uretritis, que perpetúan el ciclo de dolor y alteración de la dinámica vejiga-esfínter. El estreñimiento crónico interviene de un modo más complejo. Un recto ocupado de heces empuja a la vejiga y desvía el cuello vesical entorpeciendo el vaciado vesical; además, las heces almacenadas incrementan la actividad de la musculatura del suelo pélvico, dificultando la relajación que se requiere para la micción.

- Sociales: la dificultad para acceder al aseo o la higiene defectuosa de los baños están relacionadas con una mayor frecuencia de estreñimiento y de alteraciones miccionales en los escolares ${ }^{23,27}$.

- Por último, citar que los acontecimientos estresantes pueden provocar incontinencia o trastornos de modo secundario (es decir, una vez alcanzada la continencia y tras un período de normalidad): situación familiar conflictiva, problemas escolares, nacimiento de un hermano, cambio de cuidadores, abuso sexual, etc.

\section{Repercusión de los trastornos miccionales}

La alteración del buen funcionamiento del tracto urinario inferior puede originar: pérdidas de orina, un aumento 
de las presiones vesicales y un vaciamiento vesical incompleto (orina residual). Dependiendo del tipo de anomalía, aparecerá sólo alguna de estas consecuencias o bien una combinación de todas.

A su vez, estas tres posibles consecuencias de los trastornos funcionales pueden dar lugar a:

- Síntomas miccionales (frecuencia, manifestaciones al inicio o durante la micción, alteraciones del chorro miccional) y/o

- Deterioro del tracto urinario (el aumento de las presiones vesicales, sobre todo si hay descoordinación detrusor-esfínter, puede originar bien reflujo vesicoureteral, o más habitualmente entorpecer su resolución, o bien hipertrofia de la pared vesical, que lleva al fracaso del detrusor y a un empeoramiento de la función, derivando en una vejiga hipoactiva); y/o

- Aumento del riesgo de infección de orina, sobre todo recurrente. Cuando un paciente en edad de controlar esfínteres presenta infecciones urinarias recurrentes, hay que pensar que, con gran probabilidad, puede asociar algún trastorno miccional u otras alteraciones urológicas.

\section{Clínica: síntomas miccionales}

Las pérdidas de orina o las modificaciones del patrón miccional pueden ser el motivo de consulta pero, a menudo, los síntomas miccionales transitan inadvertidos hasta que se pregunta por ellos. El niño no los percibe como molestos (o los oculta) y pueden pasar desapercibidos para los padres o cuidadores. Por otro lado, algunos de estos síntomas pueden aparecer ocasionalmente, sin que ello signifique patología, según la edad del niño y en determinadas circunstancias (por ejemplo, tras una abundante ingesta de líquidos o retención prolongada por motivo social). Una vez alcanzada la edad de control de esfínteres hay que sospechar una alteración miccional en niños sanos cuando éstos presenten alguno de los factores que epidemiológicamente han mostrado mayor fuerza de asociación con los trastornos miccionales. Nos referimos al estreñimiento y a las infecciones de orina recurrentes. En niños con trastornos del comportamiento (fundamentalmente TDAH y trastorno de oposición desafiante) o con obesidad, también podría ser útil preguntar por la posible presencia de síntomas miccionales.

¿Cuáles son los síntomas miccionales más importantes en la infancia? Los síntomas miccionales han recibido diferentes nomenclaturas. El citado informe de 
la ICCS los clasifica de acuerdo a su relación con las fases de llenado o de vaciado de la micción ${ }^{19}$. Habitualmente estos síntomas van a estar relacionados con trastornos funcionales, pero también pueden aparecer asociados a trastornos orgánicos (tabla II). Para su evaluación hay que tener en cuenta la edad, el aporte de líquidos y el contexto clínico.

\section{Síntomas de llenado}

- Cambios en la frecuencia miccional: micciones infrecuentes o micciones frecuentes. Los niños continentes orinan de 4 a 7 veces durante el día. Ocho o más veces es una frecuencia aumentada durante el día y tres o menos veces es una frecuencia diurna disminuida. La frecuencia miccional puede aumentar de modo normal en situaciones de estrés, después de haber bebido mucho líquido y tras bebidas diuréticas (con cafeína o carbónicas).

- Incontinencia: es la pérdida incontrolable de orina de modo continuo o intermitente. Aquí nos referimos a la incontinencia intermitente y durante el día. En la nueva clasificación de síntomas no se tiene en cuenta su duración. Puede pasar desapercibida si se le escapan gotas. Se considera incontinencia aunque sólo mo- je la ropa interior o el escape sea tan pequeño que no obliga a cambiar la ropa. Lo normal es que exista sequedad completa.

- Urgencia miccional: es el deseo súbito e indemorable de orinar con temor al escape. Ha de haber control vesical para poder hablar de urgencia. El niño con urgencia miccional puede tener pérdidas; es la llamada incontinencia de urgencia. Suelen ser pequeños escapes de orina, previos a la micción, con urgencia y que se acompañan de aumento de la frecuencia miccional (necesitan saber en todo momento dónde está el baño) y de maniobras de retención para evitar los escapes. La incontinencia no es obligada. Puede no haber escapes si el esfínter externo es capaz de evitarlos. Hay que diferenciar la urgencia de la micción demorada, que es una conducta en la que el niño ignora la señal del deseo miccional hasta el último minuto, cuando está concentrado en actividades lúdicas (jugando, viendo una película o con un videojuego). La diferencia entre el síndrome de urgencia y el de micción demorada es que en el primero el niño va al aseo en cuanto puede y en el segundo no va al ba- 
ño hasta que no puede más. A pesar del deseo miccional, sigue jugando si logra retrasar la micción (en general, con disminución de la frecuencia miccional) y cuando no puede, a veces se produce un escape de orina (que suele ser importante, e incluso sigue enganchado con su actividad a pesar del escape) y otras acude rápidamente al baño.

- Nicturia: se refiere al despertar durante el sueño nocturno para orinar. Es relativamente frecuente en niños en edad escolar, no se produce más de una vez en la noche y habitualmente no indica patología. Influyen la ingesta de líquidos y el tipo de éstos (bebidas con cafeína o carbonatadas).

\section{Síntomas de vaciado}

- Vacilación (hesitancy): se refiere a la dificultad para iniciar la micción en mayores de cinco años. El niño va al baño y ha de esperar un tiempo antes de empezar a orinar. Puede ser normal, ocasionalmente, en períodos prolongados de retención (por ejemplo, cuando no pudo ir al baño por motivos escolares o sociales).

- Esfuerzo: hace referencia a que el niño usa la musculatura abdominal para iniciar y mantener la micción. Para todos los síntomas hay que tener en cuenta la edad. El esfuerzo es relevante, es decir, sugiere patología, en todos los grupos de edad.

- Chorro pobre o débil: se refiere a la emisión de un chorro de orina con poca fuerza. Este síntoma también es relevante a cualquier edad.

- Intermitencia: el chorro de orina es intermitente o en varios chorritos. Puede ser normal en menores de 3 años si no se acompaña de esfuerzo.

\section{Otros síntomas}

- Maniobras de retención: las posturas de retención son conductas aprendidas ante un deseo súbito de orinar que intentan posponer la micción o evitar la urgencia. Su propósito puede no ser reconocido por el niño, pero resulta obvio para su cuidador. Incluyen cruzar las piernas intensamente, culebreo mientras está sentado, sentarse con los muslos juntos sobre uno de sus pies, sentarse en cuclillas (squatting en inglés), reverencia de Vincent (niña que repentinamente deja lo que está haciendo, junta los muslos, se agacha cruzando las piernas y con un talón de un pie aprieta 
contra su periné), baile alrededor de un punto o compresión de los genitales con una mano.

- Sensación de vaciado incompleto: es raro que este síntoma se reconozca antes de la adolescencia.

- Goteo posmiccional: se refiere a la pérdida involuntaria de orina inmediatamente después de que la micción ha finalizado en niños con control de esfínteres maduro.

- Dolor: el dolor referido al área genital o suprapúbica se describe en adultos con alteración del tracto urinario inferior, pero no está definido en niños. La disuria sugiere infección de orina. Se interpreta que las molestias se deben al incremento de actividad muscular del suelo pélvico y del cuello vesical, así como al flujo urinario turbulento, que puede causar incomodidad uretral e incluso microhematuria ${ }^{28}$.

\section{Evaluación del problema}

Cuando sospechamos un potencial trastorno miccional, bien porque sea el motivo de consulta, o bien porque al preguntar en pacientes de riesgo (estreñimiento, ITU recurrentes, TDAH, obesidad) por la posible presencia de éste encontramos datos compatibles, ¿cuál es la evaluación diagnóstica más apropiada?
A pesar de que no hay consenso sobre el protocolo de manejo más adecuado, la valoración inicial es fundamentalmente clínica ${ }^{29,30}$. La anamnesis y una buena exploración física nos orientarán para determinar la naturaleza del problema, guiar el tratamiento y excluir causas orgánicas (neurológicas o anatómicas). También es importante el llamado diario vesical (registro de las micciones y síntomas miccionales relacionados durante la vida normal del niño mayor de cinco años). La indicación de exploraciones complementarias ha de valorarse en función de la sospecha diagnóstica. El estudio urodinámico, aunque es el patrón que nos define el tipo concreto de anomalía, en general se reserva para los casos con algún síntoma de alarma o que no responden al tratamiento habitual.

\section{Historia clínica}

La anamnesis es importante para identificar adecuadamente no sólo el problema miccional, sino también otros asociados. Hay que recoger información sobre los antecedentes y sobre todos aquellos aspectos que ayudan a delimitar el tipo de incontinencia o de trastorno y la posible existencia de anomalías asociadas (neurológicas o anatómicas). Se aconseja interrogar tanto a los padres como al niño y seguir una pauta preestablecida pa- 
ra evitar que se olviden cuestiones que pueden ser importantes (tabla III).

Siempre se ha de preguntar por el hábito intestinal. El estreñimiento es un factor que por sí solo puede causar trastor- nos miccionales o impedir su resolución. La presencia de alguno de los signos y síntomas de estreñimiento o de incontinencia fecal ha de tenerse en cuenta como una alerta sobre un posible trastorno

Tabla III. Guía para la historia clínica del niño con síntomas miccionales. Preguntas a considerar durante la anamnesis

1. Antecedentes familiares: enfermedad urológica, RVU, incontinencia, litiasis renal

2. Antecedentes personales. Control de esfínteres. ¿Enfermedad previa? (Infecciones de orina, RVU, enfermedades neurológicas congénitas 0 adquiridas)

3. Tipo de problema:

- Tiempo: ¿cuándo empezó el problema?

- Frecuencia: ¿cuántas veces sucede?

- Incontinencia: ¿moja la ropa? ¿Tiene escapes?

- ¿Intermitente/continua?

- ¿Diurna/nocturna?

- ¿Antes de orinar, inmediatamente después, entre micciones?

- ¿Síntomas con la micción? (Esfuerzo, vacilación, alteración del chorro, etc.)

4. ¿Situaciones estresantes asociadas? (Cambio de colegio, problema familiar, etc.)

5. Líquidos: ¿cuánto bebe en el día? ¿Cafeína o bebidas carbonatadas?

6. Historia miccional:

- ¿Cuántas veces orina al día? (Frecuencia miccional)

- ¿Hay urgencia?

- ¿Maniobras de retención?

- ¿Es capaz de posponer la micción si se le pide? ¿Lo hace, en situaciones concretas, para evitar ir al baño?

- ¿Duele al orinar?

- Tipo de chorro: continuo/intermitente; fuerte/débil

- ¿Se esfuerza al orinar? (Prensa abdominal)

7. Hábito intestinal:

- Dieta: líquidos, fibra

- Frecuencia de las deposiciones. ¿Estreñimiento?

- ¿Mancha la ropa interior?

- ¿Heces duras?

- ¿Episodios de dolor abdominal?

- ¿Dolor al defecar?

8. Aspectos sociales, emocionales y conductuales:

- ¿Cómo vive el problema? ¿Preocupado?

- ¿Colaborador?

- Vida escolar (integración, problemas de comportamiento)

- Vida familiar

RVU: reflujo vesicoureteral. 
miccional asociado. Por último, no se debe descuidar el área psicológica y social. Problemas en el ámbito social (sobre todo la escuela), emocional y conductual pueden influir como causa y como consecuencia. Además, es importante indagar sobre cómo afecta el problema al niño en su vida familiar y escolar.

¿Cuándo hay que sospechar causas orgánicas? En casos de aparición reciente, con un período previo de normalidad y acompañados de síntomas compatibles (por ejemplo, disuria o prurito vulvar), hay que pensar en causas irritativas (infección urinaria, vulvovaginitis, etc.). Una minoría de casos, sobre todo aquellos con síntomas de evolución prolongada y síntomas atípicos, pueden ser de etiología neurógena o anatómica. Las causas anatómicas suelen diagnosticarse en los primeros años de la vida, pero a veces pasan desapercibidas hasta la edad escolar, cuando una incontinencia continua o alteraciones del chorro miccional alertan sobre su posible existencia.

En ocasiones, las anomalías de la médula espinal no son evidentes (espina bífida oculta) y únicamente se manifiestan por incontinencia urinaria y/o fecal. Algunos autores hablan de presentación neurológica-oculta ${ }^{17}$. La espina bífida oculta es el defecto de los cuerpos verte- brales lumbosacros en la línea media (en general, en el nivel L5-S1), sin protusión de la médula espinal ni las meninges. Su prevalencia en la población general es del $1 \%$. La mayor parte de los individuos no presenta signos neurológicos y la malformación no tiene ninguna consecuencia. A veces hay marcadores cutáneos, como un mechón de pelo en la línea media de la espalda a nivel lumbosacro, un lipoma, una mancha cutánea (nevus o hemangioma), un seno dérmico o una desviación del pliegue interglúteo, que nos pueden alertar sobre la existencia de una posible espina bífida oculta. Ésta habitualmente no supone patología de la médula espinal pero, de modo excepcional, se asocia a patologías como la siringomielia, la diastematomielia y la médula anclada. Su detección precoz puede evitar el daño neurológico y las secuelas de la vejiga neurógena. Si sospechamos patología medular (tabla IV), la resonancia magnética es el método diagnóstico de elección para detectar la patología espinal ${ }^{31}$. Un estudio reciente en escolares turcos muestra una mayor probabilidad de médula anclada en aquellos con síntomas miccionales (incontinencia) $y$, dentro de este grupo, el riesgo es mayor si existen signos dermatológicos asociados o alteraciones ortopédicas o de la marcha ${ }^{32}$. 
Tabla IV. ¿Cuándo hay que sospechar una causa neurógena en los niños con incontinencia diurna?

- Historia de diabetes materna o de antecedentes familiares de espina bífida

- Signos cutáneos o anomalías en la zona media lumbosacra de la espalda: nevus, hemangioma, sinus termal o fositas, mechón de pelo

- Anomalías anorrectales (ano imperforado, atresia anal)

- Alteraciones ortopédicas (deformidades de los pies o dismetrías de las extremidades)

- Dolor en la espalda al estirar las piernas

- Incontinencia grave de heces o de orina

- Clínica miccional sugerente de neuropatía: dificultad para la micción, retención urinaria o sensación vesical débil

- Anomalías neurológicas (sensibilidad, reflejos, tono y fuerza muscular) en las extremidades inferiores y zona perineal

- Alteraciones de la marcha

- Presencia de anomalías en la radiografía de columna vertebral a nivel lumbosacro

La presencia de alguno de estos datos en la anamnesis o en la exploración física sugiere causa neurógena (patología medular) y ha de valorarse la conveniencia de solicitar una resonancia magnética espinal ${ }^{34}$.

Las válvulas de uretra posterior son la causa más frecuente de obstrucción uretral congénita (incidencia de 1/ 3.000 a 1/8.000 recién nacidos varones). Habitualmente su presencia se sospecha en la etapa prenatal (oligohidramnios, hidroureteronefrosis y distensión vesical) y el diagnóstico es temprano; pero a veces pasa desapercibida y se diagnostica más tardíamente por trastornos miccionales o por infecciones de orina. Se suponía que los casos de presentación tardía representaban un espectro más leve de la enfermedad (con menor obstrucción y mejor pronóstico a largo plazo). Sin embargo, datos recientes que comparan a niños diagnosticados después de los 5 años de edad con aquellos tratados antes de esa edad muestran grados de disfunción vesical similares en ambos grupos y peor función renal en los de diagnóstico tardío ${ }^{33}$. En estos niños de diagnóstico demorado (edad media de 7-8 años), los síntomas de presentación más frecuentes son: la incontinencia (a menudo diurna y nocturna) y los síntomas compatibles con trastorno miccional obstructivo asociado (micciones frecuentes y de poco volumen, chorro intermitente, esfuerzo, dificultad para iniciar o mantener la micción o dificultad para el vaciamiento) $)^{34,35}$. A veces hay microhematuria y leve hipertensión arterial. En la mayoría de estos niños, la ecografía vesical muestra un aumento del volumen residual posmiccional superior a los $50 \mathrm{ml}^{34}$. 


\section{Diario miccional}

El registro de las micciones y síntomas miccionales relacionados durante la vida normal del niño mayor de cinco años (diario miccional) es una herramienta fundamental para evaluar la función del tracto urinario inferior. Para resaltar su importancia, algunos autores lo clasifican dentro de los estudios urodinámicos no invasivos ${ }^{11}$. Los datos recogidos durante tres o cinco días nos permiten conocer mejor el posible trastorno; además, los padres pueden inicialmente no referir síntomas importantes (muchas veces por no haberse percatado de ellos) que sí van a reflejar en el diario.

También se han diseñado diferentes cuestionarios clínicos, como el Incontinence Symptom Index-Pediatric (ISIP) o el Pediatric Lower Urinary Tract Symptom Score (PLUTSS) para evaluar de modo numérico la incontinencia y los síntomas miccionales, estableciendo un punto de corte entre la normalidad y la enfermedad ${ }^{18,36,37}$. Sin embargo, resulta más práctico el diario vesical para una valoración inicial2 ${ }^{29}$. El diario es muy útil para perfilar el tipo de problema y ayuda en el diagnóstico diferencial, pero no es adecuado para dimensionar el trastorno. Es decir, al comparar los datos reflejados en estos diarios (volumen miccional, frecuencia e incontinencia) con los datos objetivos recogidos por personal sanitario, se ha comprobado cómo los diarios tienden tanto al hiper- como al subregistro de variables como la frecuencia miccional, el volumen miccional o los episodios de incontinencia ${ }^{38}$.

Se han usado diversos tipos de registros con diferentes nombres (diario de eliminación, carta miccional) y durante un número variable de días. La ICCS recomienda utilizar el término diario vesical y registrar durante tres o cinco días las siguientes variables ${ }^{19}$ : volumen de orina, frecuencia de micciones, pérdidas de orina (cuando y cuánto), síntomas miccionales, ingesta diaria de líquidos, episodios nocturnos (enuresis), ritmo intestinal y encopresis.

Proponemos un diario vesical en el que incluimos los puntos recomendados por la ICCS, junto con preguntas que aclaran los síntomas miccionales a los padres (tabla V). Si además hemos solicitado medir el volumen de las micciones, podemos conocer el volumen de orina medio y máximo por micción. La capacidad vesical esperada (CVE) se estima hasta los 12 años con la fórmula expresada en la tabla I y se considera que debe alcanzar los $390 \mathrm{ml}$ por encima de esa edad. El volumen miccional máximo se considera pequeño o excesivo si se encuentra, respectivamente, por 
Tabla V. Diario vesical

Registrar durante tres o cinco días los datos recogidos en la tabla que figura a continuación. Es importante anotar el número de veces que orina desde que se levanta de la cama hasta que se acuesta. También si hay problemas durante la noche. Si es posible, es muy importante medir exactamente el volumen de cada micción (pesar los pañales o utilizar una jarra con medida). Para contestar los demás apartados, tener en cuenta:

- Urgencia: no poder esperar cuando se tienen ganas de orinar, teniendo que ir al baño inmediatamente.

- Esfuerzo: tener que hacer fuerza con la tripa (como al hacer caca) para orinar.

- Maniobras de retención: posturas (como cruzar las piernas, bailotear, agacharse o llevarse la mano a los genitales) para aguantar las ganas de orinar.

- Dolor al orinar: escozor o sensación de quemazón al hacer pis. También sensación de tensión o dolor en los genitales o zona suprapúbica.

- El chorro de orina normal es rápido, continuo, sin interrupciones ni cortes, dura poco y con fuerza. Es normal que al final sea más fino.

- Para un niño son situaciones estresantes: tener un nuevo hermanito, cambio de casa, cambio de colegio, problemas escolares, problemas en casa (de salud, divorcio, muertes), abuso (sexual o psíquico), eventos especiales (cumpleaños, fiestas) u otros.

\begin{tabular}{l|l|l|l|l|l|l|l|l|l|l|l}
\hline $\begin{array}{l}\text { Nombre: } \\
\text { Fecha: }\end{array}$ & 1 & 2 & 3 & 4 & 5 & 6 & 7 & 8 & 9 & 10 & Noche \\
\hline
\end{tabular}

\section{Micciones:}

1. Hora

2. Volumen: aproximado/medido

3. ¿Moja la ropa? ¿Cuánto? ¿Antes o después de orinar?

4. ¿Síntomas asociados?

- Urgencia

- Esfuerzo

- Maniobras de retención

- Dolor

5. Chorro miccional:

- ¿Pobre o débil?

- ¿Fraccionado, irregular?

Ingesta de líquidos: ¿volumen, cafeína, refrescos?

Deposiciones:

- Hora

- ¿Molesta?

- ¿Escapes? ¿Mancha la ropa interior?

- Síntomas asociados: dolor abdominal o perianal (fisuras)

¿Alguna situación estresante? 
debajo del $65 \%$ o por encima del $150 \%$ de la CVE. Se considera poliuria la emisión, a cualquier edad, de un volumen de orina en 24 horas superior a $21 / \mathrm{m}^{2}$ de superficie corporal ${ }^{29}$.

\section{Exploración física}

Dentro de una exploración general, prestaremos atención al abdomen (masas fecales, globo vesical), a la espalda (signos de disrrafismo oculto) y a los genitales externos (irritación, estenosis meatal en niños, adherencias labiales en niñas). También es útil observar la ropa interior (¿mojada?, ¿manchada de heces?). Cuando sospechamos alteraciones en el chorro miccional, es de gran ayuda, si es posible, observar o escuchar orinar al niño (esfuerzo, chorro débil o intermitente).

La exploración ha de completarse con un examen neurológico. Se considera que la observación de la marcha sobre puntas y talones adelante y atrás puede ser la exploración neurológica básica de primer nivel ${ }^{18}$. Si es normal, podemos obviar más exploraciones. Si la anamnesis o la exploración anterior sugieren afectación neurológica, se debe realizar un estudio más completo que incluya la sensibilidad, el tono y los reflejos de las extremidades inferiores y de la región perineal (explorar también el reflejo bul- bocavernoso, ya que su ausencia sugiere lesión nerviosa sacra).

\section{Pruebas diagnósticas}

La indicación de exploraciones complementarias ha de valorarse en función de la sospecha diagnóstica. En muchos casos nos bastará un análisis de orina, un urocultivo y una ecografía abdominal; en otros serán necesarias más pruebas de imagen (cistografía, resonancia magnética) y/o estudios urodinámicos.

Aunque no hay ningún protocolo establecido, son recomendables en todos los casos el análisis de orina, el urocultivo y la ecografía renal-vesical 16,17,21. Una densidad urinaria en la primera orina de la mañana mayor de 1.020 descarta un defecto de concentración. Si la historia clínica sugiere la posibilidad de hipercalciuria (que puede manifestarse con clínica de vías urinarias bajas en aproximadamente un tercio de los casos), se ha de solicitar bioquímica sanguínea y calcio en orina ${ }^{29}$. La ecografía es una de las pruebas más útiles; realizada con la vejiga llena y posmicción, no sólo nos informa sobre los riñones y la vía urinaria superior, sino que puede excluir la obstrucción, nos estima la capacidad funcional vesical, el grosor de la pared vesical y la ausencia de residuo posmicción. Se considera que el grosor de la pared vesical normal es menor 
de $3 \mathrm{~mm}$ cuando la vejiga está llena; si es mayor de $5 \mathrm{~mm}$, puede indicar obstrucción a la salida (anatómica o por disinergia detrusor-esfínter). El volumen residual es un parámetro importante que evalúa la eficacia del vaciado vesical. Un residuo posmiccional mayor de $2 \mathrm{ml} / \mathrm{kg}$ (o mayor de $20 \mathrm{ml}$ ) es anormal. La estimación por ecografía tanto del volumen vesical como del grosor de la pared vesical es una herramienta útil para decidir a qué niños indicar estudios urodinámicos ${ }^{39,40}$.

Aunque la radiografía simple de abdomen nos puede informar de la retención fecal, no es una prueba que se considere actualmente útil para el diagnóstico de estreñimiento. Otras exploraciones han de solicitarse en función de la orientación diagnóstica y de la respuesta al tratamiento inicial. La cistografía miccional está indicada si existen engrosamiento de la pared vesical o dilatación de las vías urinarias en la ecografía, o en niños con sospecha de obstrucción (válvulas de uretra). Si hay sospecha de disrrafismo oculto, la resonancia magnética de la médula espinal es la mejor prueba para detectar posibles anomalías (en menores de seis meses es útil la ecografía de la zona lumbosacra).

\section{Estudio urodinámico}

Los estudios urodinámicos permiten evaluar la función del tracto urinario in- ferior de forma cuantificada mediante instrumentos de registro. Su desarrollo y aplicación en la infancia han permitido una mejor comprensión de las causas y un tratamiento más adecuado de los niños con trastornos miccionales. Hay diferentes técnicas que requieren un grado variable de complejidad, y algunas tienen un carácter agresivo ${ }^{29}$. Algunos autores clasifican los estudios urodinámicos en no invasivos e invasivos ${ }^{5,18}$. Dentro de los primeros, los más habituales son la uroflujometría (mide el volumen de orina evacuado por unidad de tiempo, expresado en mililitros por segundo) y la medición del volumen urinario residual posmiccional (mediante ecografía). La cistometría o cistomanometría y el estudio mediante electromiografía (EMG) del suelo pélvico pertenecen al grupo de los estudios invasivos y son las técnicas que mayor información ofrecen. El estudio urodinámico completo (que incluye al menos la medición del volumen urinario residual posmiccional, una flujometría y una EMG del suelo pélvico, y en casos seleccionados una cistomanometría) permite distinguir tres patrones urodinámicos característicos en los trastornos funcionales de la micción en la infancia ${ }^{18,21}$ : la incontinencia de urgencia (el más frecuente), la micción disfuncional y la vejiga hipoactiva o perezosa. Algunos auto- 
res añaden un cuarto tipo: la disfunción primaria del cuello vesical ${ }^{30}$.

El diagnóstico preciso de un trastorno miccional funcional no puede hacerse únicamente con criterios clínicos. Los síntomas (aunque algunos, por ejemplo la urgencia, se consideran más frecuentes en unos casos que en otros) pueden aparecer por igual en los diferentes cuadros patogénicos $y$, a veces, el mismo niño evoluciona de un cuadro a otro (por ejemplo, puede empezar con incontinencia de urgencia, continuar con micción disfuncional y acabar con una vejiga hipoactiva). La flujometría es de ayuda en la valoración hospitalaria inicial, pero con limitaciones, ya que las curvas pueden solaparse entre diferentes trastornos ${ }^{41}$. Por ello hay autores que consideran la evaluación urodinámica completa como pieza clave para una orientación diagnóstica y terapéutica adecuada de los niños con síntomas miccionales ${ }^{30}$. Pero, ¿siempre es necesario un estudio urodinámico?

El estudio urodinámico completo es invasivo, complejo, molesto y caro. El enfoque inicial de estos niños no puede incluir pruebas que se realizan en servicios especializados del nivel terciario. Una cuidadosa historia clínica, junto con la exploración física, el diario vesical y algunas pruebas bien seleccionadas pueden bas- tar para descartar (al menos inicialmente) organicidad, formular un diagnóstico de posible trastorno funcional e iniciar el tratamiento en la mayoría de los niños. Las pruebas que hay que considerar en el primer nivel de atención son el análisis de orina, el urocultivo y la ecografía renalvesical (que evalúe el volumen vesical residual $)^{42}$. Cuando el tratamiento inicial fracasa, o cuando hay sospecha de un problema orgánico (anatómico o neurógeno), es necesaria una evaluación más completa en el nivel hospitalario (preferiblemente en una unidad de urología pediátrica ${ }^{43}$. En estos casos, la mayoría de los autores opta por las pruebas urodinámicas no invasivas (diario vesical, uroflujometría y estimación del volumen vesical residual mediante ecografía) para orientar la pauta diagnóstico-terapéutica y seleccionar a los niños que precisarán un estudio urodinámico completo ${ }^{18,21}$.

\section{Trastornos funcionales del tracto urinario inferior: cuadros clínicos}

Clasificar los trastornos miccionales, especialmente aquellos cuyo síntoma más importante es la incontinencia diurna, no es una tarea fácil ${ }^{16}$. Como ya se ha mencionado, el solapamiento entre ellos es frecuente, la distinción patogénica tampoco está clara y además, con frecuencia, el mismo niño evoluciona de 
un cuadro a otro (por ejemplo, de vejiga hiperactiva a una hipoactividad vesical). Además, aunque cada uno de los cuadros clínicos presenta características que permiten diferenciarlos conceptualmente, no hay una buena correlación entre los síntomas referidos por el paciente y los datos recogidos en el diario vesical, $y$ menos aún con los resultados de las pruebas urodinámicas ${ }^{11}$. Dicho de otro modo, aunque los síntomas recogidos en la historia clínica apunten hacia un determinado patrón urodinámico (por ejemplo, vejiga hiperactiva o disfunción miccional), hasta que no se efectúa una evaluación urodinámica adecuada, no podemos estar seguros de que nuestro diagnóstico diferencial sea el correcto.

Tabla VI. Trastornos funcionales del tracto urinario inferior: cuadros clínicos con síntomas miccionales diurnos. Resumen de las características de los cuadros más importantes

\begin{tabular}{|c|c|c|c|}
\hline Nombre & Sinónimos & Características clínicas & Estudio urodinámico \\
\hline $\begin{array}{l}\text { Vejiga } \\
\text { hiperactiva } \\
\text { infantil }\end{array}$ & $\begin{array}{l}\text { Incontinencia } \\
\text { de urgencia, } \\
\text { vejiga inestable, } \\
\text { vejiga irritable }\end{array}$ & $\begin{array}{l}\text { Urgencia miccional, } \\
\text { incontinencia de urgencia } \\
\text { (pequeñas fugas), } \\
\uparrow \text { de la frecuencia miccional, } \\
\text { maniobras de retención }\end{array}$ & $\begin{array}{l}\text { Fase de llenado: } \\
\text { contracciones no inhibidas } \\
\text { del detrusor, vaciado } \\
\text { normal, no hay residuo } \\
\text { posmiccional }\end{array}$ \\
\hline $\begin{array}{l}\text { Micción } \\
\text { demorada }\end{array}$ & $\begin{array}{l}\text { Incontinencia } \\
\text { por aplazamiento } \\
\text { de la micción }\end{array}$ & $\begin{array}{l}\text { Evitan orinar, de modo voluntario y } \\
\text { habitual, en relación con situaciones } \\
\text { (juego) o lugares (colegio); } \\
\downarrow \text { frecuencia miccional; } \\
\text { incontinencia diurna si "aguanta } \\
\text { hasta que no puede más" } \\
\text { (micción completa) }\end{array}$ & Normal \\
\hline $\begin{array}{l}\text { Micción } \\
\text { disfuncional }\end{array}$ & & $\begin{array}{l}\text { Chorro urinario intermitente, } \\
\text { entrecortado (en stacatto), } \\
\text { incontinencia a veces }\end{array}$ & $\begin{array}{l}\text { Llenado normal, } \\
\text { contracción involuntaria del } \\
\text { esfínter uretral o del suelo } \\
\text { pélvico durante la micción, } \\
\text { residuo posmiccional }\end{array}$ \\
\hline $\begin{array}{l}\text { Vejiga } \\
\text { hipoactiva }\end{array}$ & Vejiga perezosa & $\begin{array}{l}\downarrow \text { frecuencia miccional; } \\
\text { usan la prensa abdominal } \\
\text { para orinar; chorro urinario débil, } \\
\text { a veces fraccionado }\end{array}$ & $\begin{array}{l}\text { Alteraciones de llenado } \\
\text { y de vaciado, detrusor } \\
\text { hipoactivo, residuo } \\
\text { posmiccional, } \\
\uparrow \text { capacidad vesical }\end{array}$ \\
\hline $\begin{array}{l}\text { Reflujo } \\
\text { vaginal }\end{array}$ & Micción vaginal & $\begin{array}{l}\text { Escapes de orina de pequeño } \\
\text { volumen, al ponerse de pie, } \\
\text { siguiendo a una micción normal } \\
\text { y sin asociar ningún otro síntoma } \\
\text { de vías urinarias bajas }\end{array}$ & $\begin{array}{l}\text { Normal, } \\
\text { reflujo de orina hacia la } \\
\text { vagina por adherencias } \\
\text { labiales o bien por no } \\
\text { separar suficientemente la } \\
\text { piernas durante la micción }\end{array}$ \\
\hline
\end{tabular}


No obstante, a pesar de que su utilidad, desde un punto de vista asistencial, está en entredicho, aún se mantiene la clasificación de los trastornos miccionales funcionales en cuadros clínicos ${ }^{31}$. En el documento de la ICCS de 2006 se distinguen, por un lado, la enuresis (entendida como incontinencia nocturna) $y$, por otro, los cuadros o condiciones con síntomas diurnos (tabla VI) ${ }^{16}$. Una vez más, los límites no son absolutos: por un lado, un porcentaje importante de niños con enuresis puede asociar otros síntomas miccionales diurnos; y, por otro, en ocasiones, los niños que consultan por incontinencia intermitente durante el día tienen pérdidas tanto diurnas como nocturnas.

\section{Tratamiento de la incontinencia y los trastornos miccionales}

\section{Uroterapia estándar}

Aunque la orientación terapéutica puede variar en función del patrón urodinámico, el manejo inicial de los niños con algún trastorno miccional es fundamentalmente clínico ${ }^{17,18}$. Si no hay sospecha de patología orgánica, el tratamiento básico de un niño con síntomas miccionales combina una serie de intervenciones que algunos autores resumen bajo el nombre de uroterapia estándar o rehabilitación micciona ${ }^{29,41}$. Dicha uroterapia incluye:

- Comprensión del problema tanto por el niño como por su familia. Hay que explicar la función normal del tracto urinario inferior y las posibles alteraciones que influyen en el trastorno del paciente.

- Modificaciones dietéticas. Eliminar la cafeína (estimulante y diurético) y las bebidas carbonatadas. Aconsejar una buena hidratación a través de una ingesta regular de líquidos (fundamentalmente agua) y aumentar la fibra en la dieta.

- Tratamiento del estreñimiento. Es esencial combatir el estreñimiento. Con frecuencia, si conseguimos un hábito intestinal regular $y$, lo que es más importante, una deposición diaria sin molestias (hay niños con una deposición diaria molesta que acumulan heces), el problema miccional disminuye o desaparece.

- Higiene genital y perineal adecuada (para evitar irritaciones). Proponer el lavado diario de perineo y genitales de modo correcto (de delante hacia atrás en niñas, retracción de prepucio, etc.) y evitando tanto jabones irritantes como baños prolongados.

- Programa miccional: 
- Postura al orinar: se aconseja que las niñas se sienten con los pies apoyados, la espalda recta (leve inclinación hacia delante) y que separaren bien las piernas al orinar (a veces, la forma más efectiva de conseguirlo es hacer que la niña se siente de espaldas al váter mientras orina). En niños también sería aconsejable orinar sentados (se favorece la relajación del suelo pélvico). No se aconseja el uso de orinales.

- Orinar con frecuencia: cada 2-3 horas (6-7 micciones al día). Siempre al acostarse y al levantarse. El niño debe entender que debe orinar según esta pauta regular para evitar la urgencia. Al corregir la urgencia, también se evita que utilicen maniobras de retención (con contracción voluntaria del esfínter). En niños que orinan infrecuentemente puede ser más realista pedirles que orinen cada 3-4 horas. Es útil un diario miccional donde anotar los progresos.

- Orinar sin prisas y completamente (hasta vaciar la vejiga). Contar hasta 10 ó 15 antes de subirse la cremallera o levantarse del inodoro.
Junto con estas medidas estándar, hay que prestar atención al tratamiento de los posibles problemas psicopatológicos asociados, como el TDAH, la depresión, la ansiedad o el trastorno de oposicióndesafiante. También puede ser útil el control del peso, ya que, como se mencionó, se ha relacionado la obesidad con una peor respuesta al tratamiento en niños con enuresis nocturna o con trastornos miccionales ${ }^{26}$.

\section{Tratamiento farmacológico}

Cuando el tratamiento inicial fracasa es conveniente reevaluar al paciente. Hay que asegurar un buen cumplimiento del diario vesical y la normalidad de la ecografía (evaluando riñón, vías urinarias, vejiga y la ausencia de residuo posmiccional). Si se descarta patología orgánica, no hay historia de ITU febril previa y la orientación diagnóstica apunta hacia una vejiga hiperactiva, puede pautarse un tratamiento combinado (anticolinérgicos junto con pautas estándar) ${ }^{44}$. No obstante, varios autores aconsejan considerar la opción de derivar hacia las consultas hospitalarias (preferentemente a urología pediátrica) cuando falla la respuesta a la terapia básica o estándar ${ }^{17,18}$.

En la vejiga hiperactiva, los anticolinérgicos pueden ayudar a disminuir las contracciones vesicales no inhibidas y a 
aumentar la capacidad vesical. La oxibutinina (Ditropan ${ }^{\circledR}$ ) es uno de los más empleados, pero con cierta frecuencia puede producir efectos secundarios (sequedad de piel, boca y ojos, dilatación de pupilas, estreñimiento, cefalea, cambios de humor, somnolencia, intolerancia al calor, pérdida de apetito, posibilidad de retención urinaria y riesgo de infección de orina por la orina residual). La oxibutinina se recomienda dos o tres veces al día $(0,1-0,15 \mathrm{mg} / \mathrm{kg}$ por dosis).

La tolterodina (Detrusitol ${ }^{\circledR}$, Urotrol ${ }^{\circledR}$ ) es un fármaco de comercialización más reciente con mejor tolerancia y, aunque no consta indicación pediátrica en su ficha técnica, podría considerarse el anticolinérgico de elección ${ }^{44-46}$. La dosis inicial aconsejada es de $1 \mathrm{mg}$ cada 12 horas $(0,02 \mathrm{mg} / \mathrm{kg}$ cada $12 \mathrm{~h})$ y puede incrementarse hasta 2 mg cada 12 horas. Hay una formulación de efecto prolongado (Urotrol $\mathrm{Neo}^{\oplus}$ ) que permite su administración en una dosis única diaria (1-2 mg cada 24 horas).

\section{Otras medidas}

Cuando las medidas habituales no tienen éxito, hay que contemplar otras opciones terapéuticas. La etiología multifactorial de los trastornos miccionales funcionales obliga a tener en cuenta di- ferentes posibilidades, no sólo el tratamiento con anticolinérgicos ${ }^{28}$. Se incluyen diferentes medidas específicas que corresponden al nivel hospitalario y que esbozamos a continuación:

- Si se produce un aumento del residuo posmiccional, pueden usarse alfabloqueantes (tamsulosina, doxazosina, terazosina) para mejorar la disfunción del cuello vesical o la disinergia del esfínter uretral externo, relajar el cuello vesical y la uretra posterior. Son fármacos sin autorización pediátrica y que pueden ocasionar efectos secundarios, por ejemplo, hipotensión.

- Cateterización vesical simple: en casos seleccionados, puede estar indicado el vaciado regular de la vejiga mediante sondaje vesical realizado por el propio paciente o sus familiares con el fin de evitar que se acumule orina residual.

- Biofeedback: es una forma de reeducación en la que el paciente se hace consciente de un proceso fisiológico (de tipo neuromuscular, como la micción) y que hasta ese momento realiza de forma autónoma9. Para lograr la percepción del proceso se usan habitualmente sistemas computarizados, que, con la ayuda de la EMG, transfor- 
man la contracción muscular en señales visuales, auditivas o táctiles, que el paciente identificará fácilmente (por ejemplo, al contraer el esfínter aparece un pez de color en la pantalla). En el biofeedback urinario se intenta modular la respuesta de la musculatura pélvica durante las fases miccionales de llenado y vaciado.

- Agonistas de la serotonina (tega- serod): este grupo de fármacos puede ser útil al mejorar la motilidad intestinal y el estreñimiento. Parece que también disminuyen la hiperactividad del detrusor (su acción en el SNC puede influir sobre las vías de regulación) ${ }^{28}$.

- Otras opciones experimentales: toxina botulínica $A$, estimulación de la médula espinal o de los nervios periféricos ${ }^{28,41}$.

\section{Bibliografía}

1. Shaikh $N$, Hoberman A, Wise B, Kurs-Lasky $M$, Kearney $D$, Naylor $S$, et al. Dysfunctional Elimination Syndrome: Is It Related to Urinary Tract Infection or Vesicoureteral Reflux Diagnosed Early in Life? Pediatrics. 2003;112:1134-7.

2. Hellström AL, Hanson E, Hansson $S$, Hjälmas $\mathrm{K}$, Jodal $\mathrm{U}$. Micturition habits and incontinence in 7-year-old Swedish school entrants. Eur J Pediatr. 1990;149(6):434-7.

3. Kajiwara $M$, Inoue $K$, Usui $A$, Kurihara $M$, Usui T. The micturition habits and prevalence of daytime urinary incontinence in Japanese primary school children. J Urol. 2004;171(1):403-7.

4. Hellström $A$, Hanson $E$, Hansson $S$, Hjälmas $\mathrm{K}$, Jodal $\mathrm{U}$. Micturition habits and incontinence at age 17: reinvestigation of a cohort studied at age 7. Br J Urol. 1995;76(2):231-4.

5. Feldman A, Bauer SB. Diagnosis and management of dysfunctional voiding. Curr Opin Pediatr. 2006;18(2):139-47.

6. Halachami S, Farhat WA. Interactions of constipation, dysfunctional elimination syndrome and vesicoureteral reflux. Adv Urol. 2008:828 275.

7. Loening-Baucke V. Prevalence rates for constipation and faecal and urinary incontinence. Arch Dis Child. 2007;92:486-9.

8. Hellström A, Hanson $E$, Hansson S, Hjälmas $\mathrm{K}$, Jodal $\mathrm{U}$. Association between urinary symptoms at 7 years old and previous urinary tract infection. Arch Dis Child. 1991;66(2):232-4.

9. Blanco JL, Oliver FJ, De Celis R, Joao CM. Tratamiento de los trastornos miccionales en niños mediante biofeedback. Cir Pediatr. 2006;19(2): 61-5.

10. National Collaborating Centre for Women's and Children's Health. Urinary tract infection in children: diagnosis, treatment and long-term management. National Institute for Health and Clinical Excellence (NICE). August 2007 [consultado el 20/10/2009]. Disponible en www.nice.org.uk

11. Barroso U, Nova T, Dultra A, Lordelo P, Andrade J, Vinhaes AJ. Comparative analysis of the symptomatology of children with lower urinary tract dysfunction in relation to objetive data. Int Braz J Urol. 2006;32(1):70-6. 
12. Ural Z, Ulman I, Avanoglu A. Bladder dynamics and vesicoureteral reflux: factors associated with idiopathic lower urinary tract dysfunction in children. J Urol. 2008;179:1564-7.

13. Yeung CK, Sreedhar B, Sihoe JD, Sit FK. Renal and bladder functional status at diagnosis as predictive factors for the outcome of primary vesicoureteral reflux in children. J Urol. 2006;176(3): 1152-6.

14. Snodgrass W. The impact of treated dysfunctional voiding on the nonsurgical management of vesicoureteral reflux. J Urol. 1998;160(5): 1823-5.

15. Upadhyay J, Bolduc S, Bagli DJ, McLorie $G A$, Khoury $A E$, Farhat $W$. Use of the dysfunctional voiding symptom score to predict resolution of vesicoureteral reflux in children with voiding dysfunction. J Urol. 2003;169(5):1842-6.

16. Herndon $C D$, Joseph $D B$. Urinary incontinence. Pediatr Clin North Am. 2006;53(3):363-77.

17. Schulman SL, Berry AK. Helping the child with daytime wetting stay dry. Contemporary Pediatrics. Jun. 2006 [consultado el 20/10/ 2009]. Disponible en www.contemporarypediatrics.com

18. Úbeda Sansano MI, Martínez García R, Díez Domingo J. Guía de práctica clínica: enuresis nocturna primaria monosintomática en Atención Primaria. Rev Pediatr Aten Primaria. 2005;7(supl 3): s77-s78.

19. Neveus T, Von Gontard A, Hoebeke P, Hjalmas K. The standardization of terminology of lower urinary tract function in children and adolescents: report from the Standardisation Committee of the international Children's Continence Society. J Urol. 2006;176(1):314-24.

20. Joinson C, Heron J, Gontard A and the ALSPAC Study Team. Psychological problems in children with daytime wetting. Pediatrics. 2006;118 (5):1985-93.
21. Hjälmas K, Hoebeke PB, Paepe H. Lower urinary tract dysfunction and urodynamics in children. Eur Urol. 2000;38(5):655-65.

22. Franco I. Overactive bladder in children. Part 1: Pathophysiology. J Urol. 2007;178(3 Pt 1):7618.

23. Chung JM, Lee SD, Kang DI, Kwon DD, Kim KS, Kim SY, et al. Prevalence and associated factors of overactive bladder in Korean children 5-13 years old: a nationwide multicenter study. Urol. 2009:73 (1):63-7.

24. Zink S, Freitag CM, Gontard A. Behavioral comorbidity differs in subtypes of enuresis and urinary incontinence. J Urol. 2008;179:295-8.

25. Erdem E, Lin A, Kogan BA, Feustel PJ. Association of elimination dysfunction and body mass index. J Pediatr Urol. 2006;2(4):364-7.

26. Guven A, Giramonti K, Kogan BA. The effect of obesity on treatment efficacy in children with nocturnal enuresis and voiding dysfunction. $J$ Urol. 2007;178:1458-62.

27. Kistner M. Dysfunctional elimination behaviors and associated complications in school-age children. J Sch Nurs. 2009;25(2):108-16.

28. Franco I. Overactive bladder in children. Part 2: Management. J Urol. 2007;178(3 Pt 1):769-74.

29. Fernández $M$, Rodríguez LM. Trastornos miccionales en la infancia. Protocolos de Nefrología de la AEP 2008 [consultado el 25/05/2009]. Disponible en www.aeped.es/protocolos/nefro/ index.htm

30. Glassberg KI. Evaluating children with nonneurogenic voiding disorders. Can we do better? J Urol. 2008;180(4):1226-7.

31. Wraige $E$, Borzyskowski M. Investigation of daytime wetting: when is spinal cord imaging indicated? Arch Dis Child. 2002;87(2):151-5.

32. Bademci G, Saygun M, Batay F, Cakmak A, Basar $H$, Anbarci $H$, et al. Prevalence of primary tethered cord syndrome associated with occult spi- 
nal dysraphism in primary school children in Turkey. Pediatr Neurosurg. 2006;42(1):4-13.

33. Ziylan O, Optar T, Ander H, Korgali E, Rodoplu $\mathrm{H}$, Kocak $\mathrm{T}$. The impact of the late presentation of posterior urethral valves on bladder and renal function. J Urol. 2006;175(6 Pt1):1894-7.

34. Schober JM, Dulabon LM, Woodhouse CR. Outcome of valve ablation in late-presenting posterior urethral valves. BJU Int. 2004;94(4):616-9.

35. Warren J, Pike JG, Leonard MP. Posterior urethral valves in Eastern Ontario: a 30 year perspective. Can J Urol. 2004;11(2):2210-5.

36. Nelson CP, Park JM, Bloom DA, Wan J, Dunn RL, Wei JT. Incontinence Symptom IndexPediatric: development and initial validation of a urinary incontinence instrument for the older pediatric population. J Urol. 2007;178(4 Pt 2):1763-7.

37. Dogan HS, Akpinar B, Gurocak S, Akata D, Bakkaloglu M, Tekgul S. Non-invasive evaluation of voiding function in asymptomatic primary school children. Pediatr Nephrol. 2008;23(7):1115-22.

38. Bael AM, Lax H, Hirche $H$, Gäbel E, Winkler $P$, Hellström $A L$, et al. Self-reported urinary incontinence, voiding frequency, voided volume and pad-test results: variables in a prospective study in children. BJU Int. 2007;100(3):651-6.

39. Kelly CE. Evaluation of voiding dysfunction and measurement of bladder volume. Rev Urol. 2004;6 (Supl 1):s32-s37.
40. Yeung CK, Sreedhar B, Leung Y, Sit K. Correlation between ultrasonographic bladder measurements and urodynamic findings in children with recurrent urinary tract infection. BJU Int. 2007;99(3):651-5.

41. Glassberg KI, Combs AJ. Nonneurogenic voiding disorders: what's new? Curr Opin Urol. 2009;19(4):412-8.

42. Izquierdo RM, Luque Mialdea R. Evaluation of the lower urinary tract function in pediatric patients with primary vesicoureteral reflux. Arch Esp Urol. 2008;61(2):191-207.

43. Bael A, Lax $H$, De Jong TP, Hoebeke P, Nijman $R J$, Sixt $R$, et al. The relevance of urodynamic studies for Urge syndrome and dysfunctional voiding: a multicenter controlled trial in children. J Urol. 2008;180(4):1486-93.

44. Ayan S, Topsakal K, Gokce G, Gultekin EY. Efficacy of combined anticholinergic treatment and behavioral modification as a first line treatment for nonneurogenic and nonanatomical voiding dysfunction in children: a randomized controlled trial. J Urol. 2007;177(6):2325-9.

45. Babu R. Effectiveness of tolterodine in nonneurogenic voiding dysfunction. Indian Pediatr. 2006;43(11):980-3.

46. Salvatore $S$, Serati M, Bolis P. Tolterodine for the treatment of overactive bladder. Expert Opin Pharmacother. 2008;9(7):1249-55. 
